University of New Orleans

ScholarWorks@UNO

$9-10-2009$

\title{
Broadband IR polarizing beam splitter using a subwavelength- structured one-dimensional photonic-crystal layer embedded in a high-index prism
}

\author{
R. M.A. Azzam \\ University of New Orleans, razzam@uno.edu \\ H. K. Khanfar
}

Follow this and additional works at: https://scholarworks.uno.edu/ee_facpubs

Part of the Electrical and Electronics Commons

\section{Recommended Citation}

H. K. Khanfar and R. M. A. Azzam, "Broadband IR polarizing beam splitter using a subwavelengthstructured one-dimensional photonic-crystal layer embedded in a high-index prism," Appl. Opt. 48, 5121-5126 (2009).

This Article is brought to you for free and open access by the Department of Electrical Engineering at ScholarWorks@UNO. It has been accepted for inclusion in Electrical Engineering Faculty Publications by an authorized administrator of ScholarWorks@UNO. For more information, please contact scholarworks@uno.edu. 


\title{
Broadband IR polarizing beam splitter using a subwavelength-structured one-dimensional photonic-crystal layer embedded in a high-index prism
}

\author{
H. K. Khanfar and R. M. A. Azzam* \\ Department of Electrical Engineering, University of New Orleans, New Orleans, Louisiana 70148, USA \\ ${ }^{*}$ Corresponding author razzam@uno.edu
}

Received 16 July 2009; revised 22 August 2009; accepted 24 August 2009; posted 25 August 2009 (Doc. ID 114367); published 10 September 2009

\begin{abstract}
An iterative procedure for the design of a polarizing beam splitter (PBS) that uses a form-birefringent, subwavelength-structured, one-dimensional photonic-crystal layer (SWS 1-D PCL) embedded in a highindex cubical prism is presented. The PBS is based on index matching and total transmission for the $p$ polarization and total internal reflection for the $s$ polarization at the prism-PCL interface at $45^{\circ}$ angle of incidence. A high extinction ratio in reflection $(>50 \mathrm{~dB})$ over the $4-12 \mu \mathrm{m}$ IR spectral range is achieved using a SWS 1-D PCL of ZnTe embedded in a ZnS cube within an external field of view of $\pm 6.6^{\circ}$ and in the presence of grating filling factor errors of up to $\pm 10 \%$. Comparable results, but with wider field of view, are also obtained with a Ge PCL embedded in a Si prism. (C) 2009 Optical Society of America

OCIS codes: $\quad 050.2770,230.1360,260.5430,310.1620$.
\end{abstract}

\section{Introduction}

Polarizing beam splitters (PBSs) are used to separate an incident light beam into two beams with orthogonal linear polarizations (often denoted by $p$ and $s$ ) that travel in different directions. Conventional PBSs employ bulk crystal optics [1] or multilayer interference coatings that are embedded in a prism [2-6]. More recently, PBSs that use diffraction gratings [7-9] and photonic crystals [10,11] have also been introduced.

In this paper a PBS is described that uses the form birefringence of subwavelength-structured (SWS) one-dimensional photonic-crystal layer (1-D PCL) embedded in a high-index cubical prism. The nondiffracting SWS 1-D PCL acts as a homogeneous uniaxial crystal whose optic axis is oriented perpendicular to the plane of incidence. At the prism-PCL interface the $p$ polarization is totally transmitted and the $s$

0003-6935/09/275121-06\$15.00/0

(C) 2009 Optical Society of America polarization is totally internally reflected at $45^{\circ}$ angle of incidence.

In Section 2 an iterative design procedure is described that initially ignores light interference in the PCL. In Section 3 a broadband $(4-12 \mu \mathrm{m})$ IR PBS that uses a ZnTe 1-D PCL sandwiched between two $\mathrm{ZnS}$ right-angle isosceles prisms is demonstrated. Such a device performs well within an external field of view (FOV) of $\pm 6.6^{\circ}$ and is reasonably insensitive to small $( \pm 10 \%)$ changes of the grating region filling factor. In Section 4 , interference in the PCL is considered, and its effect on the extinction ratios of the PBS in reflection and transmission is analyzed. Section 5 presents additional results for an IR PBS that uses a Ge PCL embedded in a Si prism. Finally some concluding remarks are given in Section $\underline{6}$.

\section{Basic Design Procedure}

Figure 1(a) shows a cross section of the 1-D PCL of thickness $d$ that consists of a rectangular grating of coating material of refractive index $n_{c}$, period $\Lambda$, 
and filling factor $f$. The layer is deposited on an optically isotropic substrate (prism) of refractive index $n$, and the gaps between grating ridges are assumed to be filled with air or an inert gas of refractive index 1 . Figure $1(\mathrm{~b})$ shows the PBS cube with a grating vector $\vec{K}_{G}$ of the PCL normal to the plane of incidence. $p$ and $s$ denote the linear polarizations parallel and perpendicular to the plane of incidence, respectively.

With the grating period $\Lambda \ll \lambda$, where $\lambda$ is the vacuum wavelength of incident light, the SWS 1-D PCL is equivalent to a nondiffracting homogenous uniaxial crystal layer with its optic axis parallel to the grating vector. The second-order ordinary (o) and extraordinary $(e)$ refractive indices of such a layer are given by [12]

$$
\begin{gathered}
n_{o}^{(2)}=\left\{\left(n_{o}^{(1)}\right)^{2}+\frac{1}{3}\left[\pi \frac{\Lambda}{\lambda} f(1-f)\right]^{2}\left(n_{c}^{2}-1\right)^{2}\right\}^{1 / 2} \\
n_{e}^{(2)}=\left\{\left(n_{e}^{(1)}\right)^{2}+\frac{1}{3}\left[\pi \frac{\Lambda}{\lambda} f(1-f)\right]^{2}\right. \\
\left.\times\left(\frac{1}{n_{c}^{2}}-1\right)^{2}\left(n_{e}^{(1)}\right)^{6}\left(n_{o}^{(1)}\right)^{2}\right\}^{1 / 2}
\end{gathered}
$$

in which

$$
\begin{aligned}
& n_{o}^{(1)}=\left(1-f+n_{c}^{2} f\right)^{1 / 2}, \\
& n_{e}^{(1)}=\left(1-f+f / n_{c}^{2}\right)^{-1 / 2},
\end{aligned}
$$

are the first-order indices that are independent of $\Lambda / \lambda$.

With the grating vector (optic axis) normal to the plane of incidence, Fig. 1(b), p-polarized light sees the ordinary index, $n_{o}=\overline{n_{0}^{(2)}}$ [the superscript (2) is dropped for simplicity], and is totally transmitted at the prism-PCL interface at all angles of incidence when index matching is satisfied, i.e.,

$$
n_{o}=n
$$

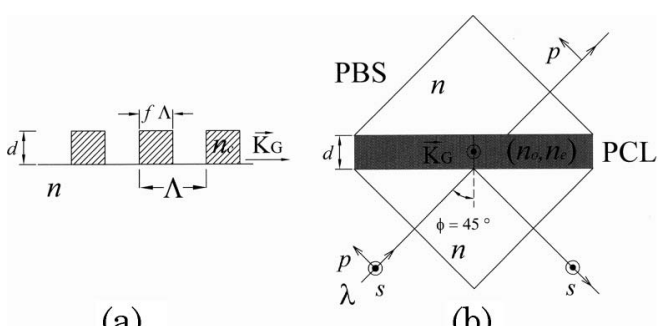

(a)

(b)

Fig. 1. (a) Cross section of 1-D PCL of thickness $d$ that consists of rectangular grating of a coating material of refractive index $n_{c}$, period $\Lambda$, and filling factor $f$. The layer is deposited on an optically isotropic substrate (prism) of refractive index $n$. (b) Cube PBS using an embedded PCL with grating vector $\vec{K}_{G}$ normal to the plane of incidence. $p$ and $s$ denote the linear polarizations parallel and perpendicular to the plane of incidence, respectively.
On the other hand, the $s$ polarization, which is parallel to the optic axis, sees the extraordinary index $n_{e}=n_{e}^{(2)}$ and experiences total internal reflection if the critical angle $\phi_{c s}$ is $<45^{\circ}$. Equivalently, we write

$$
\begin{aligned}
\phi_{c s} & =\sin ^{-1} x, \\
x & =n_{e} / n<1 / \sqrt{2} .
\end{aligned}
$$

To avoid significant tunneling of $s$-polarized light across the PCL and to achieve high extinction ratio in transmission, the PCL thickness $d$ must be much greater than the penetration depth of the evanescent $s$ wave in the PCL. This condition is assumed to hold, and interference effects in the PCL are ignored in this section.

From Eqs. (4) and (5) the following relation between the ordinary and extraordinary refractive indices of the PCL is obtained:

$$
n_{e}=x n_{o} .
$$

By substituting $n_{o}$ and $n_{e}$ from Eqs. (1)-(3) in Eq. (6), one key equation in the three design parameters $x, n_{c}$, and $f$ is obtained:

$$
\begin{aligned}
x^{2}\left\{\left(1-f+n_{c}^{2} f\right)+\right. & \left.\frac{1}{3}\left(\pi \frac{\Lambda}{\lambda_{0}} f(1-f)\right)^{2}\left(n_{c}^{2}-1\right)^{2}\right\} \\
= & \left(1-f+f / n_{c}^{2}\right)^{-1}+\frac{1}{3}\left(\pi \frac{\Lambda}{\lambda_{0}} f(1-f)\right)^{2} \\
& \times\left(\frac{1}{n_{c}^{2}}-1\right)^{2}\left(1-f+f / n_{c}^{2}\right)^{-3} \\
& \times\left(1-f+n_{c}^{2} f\right) .
\end{aligned}
$$

For a given operating wavelength $\lambda$ and grating period $\Lambda$, the design proceeds according to the following steps:

1. $n_{c}$ of a suitable transparent coating material and an initial value of $x<0.707$ are assumed, and Eq. (7) is solved for $f$.

2. $f$ is substituted back in Eqs. (1) -(3) to obtain $n_{o}$ and $n_{e}$ of the PCL.

3. Equation (4) is used to determine the refractive index $n$ of the prism and to verify that $n<n_{c}$.

4. If $n$ does not match the refractive index of a suitable preselected transparent substrate, $x$ is adjusted, and steps (1)-(3) are repeated until $n$ equals the refractive index of the prism material at the design wavelength to within 4 decimal places.

\section{Polarizing Beam Splitter Using ZnTe Photonic- Crystal Layer Embedded in ZnS Cube}

A PBS that uses an IR-transparent ZnTe PCL, which is sandwiched between two right-angle $\mathrm{ZnS}$ prisms, is considered for the $\mathrm{CO}_{2}$-laser wavelength $\lambda=10.6 \mu \mathrm{m}$. A grating period of $\Lambda=1 \mu \mathrm{m}$ and $n_{c}(\mathrm{ZnTe})=2.6818$ [13] are assumed. By applying the iterative procedure of Section $\underline{2}$, we find that at $\phi_{c s}=41.8314^{\circ}$, Eq. (ㅁ) 
yields $f=0.6042$. From Eqs. (1) $-(3), n_{o}=2.1921$ and $n_{e}=1.4620$ are obtained. Thus the PCL has large negative birefringence $\Delta n=n_{e}-n_{o}=-0.73$. From the index-matching condition, Eq. (4), we have $n=2.1921$, which coincides with the refractive index of $\mathrm{ZnS}$ at $\lambda=10.6 \mu \mathrm{m} \mathrm{[13].} \mathrm{Therefore} \mathrm{a} \mathrm{self-consistent}$ solution to the PBS design is obtained, with the device parameters summarized in Table 1 .

An important parameter that characterizes the performance of the PBS is its reflection extinction ratio, which is defined by

$$
\mathrm{ER}_{r}=10 \log _{10}\left(R_{s} / R_{p}\right) .
$$

The reflection coefficients of the prism-PCL interface for incident $p$ - and $s$-polarized light are calculated using expressions that are given in [14]. In Fig. 2 the intensity reflectance $R_{p}$ of the prismPCL interface for the $p$ polarization and the extinction ratio in reflection $\mathrm{ER}_{r}$ [Eq. (8)] are plotted versus the filling factor $f$ as it is changed by approximately $\pm 10 \%$ around the design value of 0.604 , with all other parameters kept constant. Total internal reflection for the $s$ polarization $\left(R_{s}=1\right)$ is maintained over this range of $f$. Interference effects in the PCL are initially ignored; these effects are considered later in Section 4. Figure 2 shows that an $\mathrm{ER}_{r}>50 \mathrm{~dB}$ is obtained for $0.554 \leq f \leq 0.654$, which indicates good tolerance to small dimensional errors.

Figure 3 presents the deviation from index matching of $p$-polarized light $\left(n_{o}=n\right)$ as $\lambda$ is varied over the IR range $4 \leq \lambda \leq 12 \mu \mathrm{m}$, while all other parameters are kept constant at their design values. It is evident from Fig. 3 that index matching is satisfied at $\lambda=$ $10.6 \mu \mathrm{m}$ and also occurs coincidentally at $\lambda=$ $5.55 \mu \mathrm{m}$, which is approximately half of the design wavelength. Over the entire bandwidth the dispersion of the coating and prism materials is accounted for [13], and total internal reflection for the $s$ polarization is maintained.



Fig. 2. (Color online) Intensity reflectance $R_{p}$ and extinction ratio $\mathrm{ER}_{r}(\mathrm{~dB})$ of prism-PCL interface are plotted as functions of grating filling factor $f$. These calculations are for ZnTe 1-D PCL embedded in $\mathrm{ZnS}$ prism, with grating period $\Lambda=1 \mu \mathrm{m}$, wavelength $\lambda=10.6 \mu \mathrm{m}$, and $45^{\circ}$ angle of incidence.

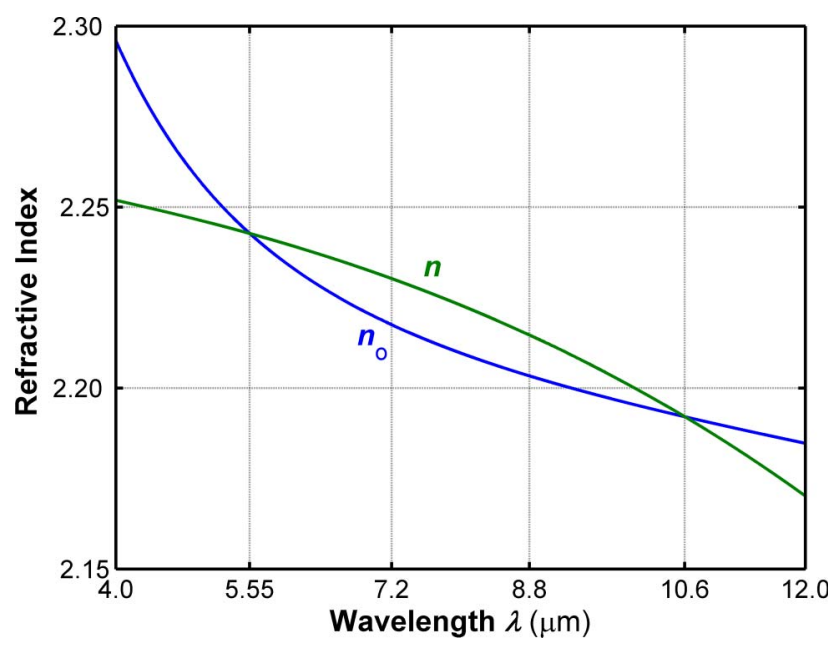

Fig. 3. (Color online) Deviation from index matching of $p$ polarized light (i.e., $n_{o}=n$ ) as the wavelength $\lambda$ is varied over the IR spectral range $4 \leq \lambda \leq 12 \mu \mathrm{m}$. These calculations assume ZnTe 1-D PCL embedded in ZnS prism with grating period $\Lambda=1 \mu \mathrm{m}$, and they take into account dispersion of the optical properties of $\mathrm{ZnTe}$ and $\mathrm{ZnS}$.

Figure 4 shows $R_{p}$ and $\mathrm{ER}_{r}$ plotted versus wavelength $\bar{\lambda}$, for $4 \leq \lambda \leq 12 \mu \mathrm{m}, \quad \Lambda=1 \mu \mathrm{m}$, and $f=0.6042$. Notice that the $\mathrm{ER}_{r}$ is $>70 \mathrm{~dB}$ over the full spectral range and has sharp peaks at $\lambda=5.55$ and $10.6 \mu \mathrm{m}$. Therefore near-ideal broadband polarization on reflection is possible.

Because index matching for the $p$ polarization is independent of angle of incidence $\phi$, the PBS cube performs well within a limited FOV as long as $\phi>$ $\phi_{c s}$. Given that $\phi_{c s} \approx 41.83^{\circ}$ (Table 1 ) the internal FOV in the plane of incidence in Fig. $1(\mathrm{~b})$ is restricted to about $3^{\circ}$ below the nominal operating angle $\phi=45^{\circ}$. The corresponding range of near-normal incidence angles at the air-prism interface is $n \times 3^{\circ}=$ $6.6^{\circ}$ according to Snell's law. The FOV is considerably larger at high angles of incidence, $\phi>45^{\circ}$.

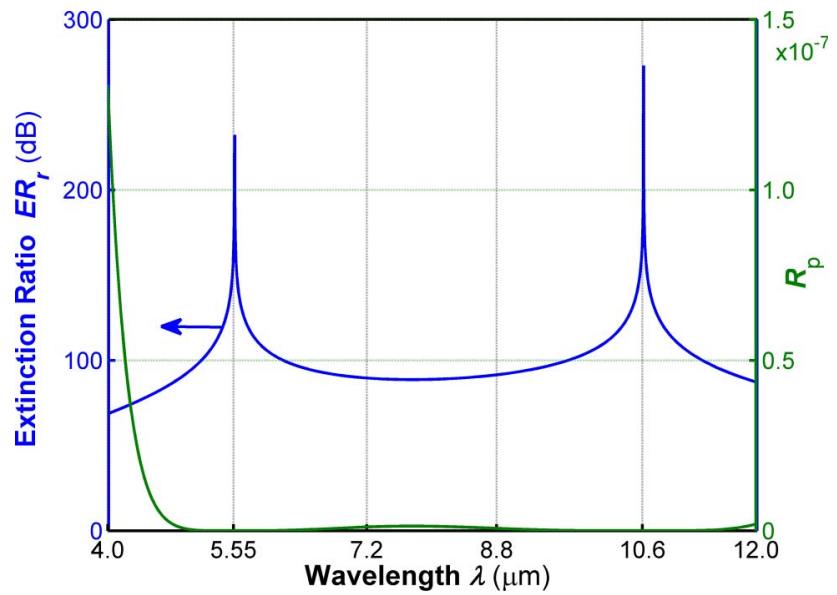

Fig. 4. (Color online) Intensity reflectance $R_{p}$ and extinction ratio $\mathrm{ER}_{r}$ plotted versus wavelength $\lambda, 4 \leq \lambda \leq 12 \mu \mathrm{m}$, for a PBS that uses ZnTe 1-D PCL embedded in ZnS prism with grating period $\Lambda=$ $1 \lambda \mathrm{m}$ and filling factor $f=0.604$. Dispersion of prism and coating materials is accounted for. 
Table 1. Design Parameters for PBS Using ZnTe PCL with $\Lambda=1 \mu \mathrm{m}$ Embedded in $\mathrm{ZnS}$ Cube at $10.6 \mu \mathrm{m}$ Wavelength

\begin{tabular}{cccccc}
\hline$n_{c}(\mathrm{ZnTe})$ & $f$ & $n_{e}$ & $n_{o}$ & $n(\mathrm{ZnS})$ & $\phi_{c s}$ \\
\hline 2.6818 & 0.6042 & 1.4620 & 2.1921 & 2.1921 & $41.8314^{\circ}$ \\
\hline
\end{tabular}

\section{Interference Effects within the Photonic-Crystal Layer}

In previous sections the PCL thickness $d$ is assumed to be sufficiently large, and interference effects within the layer are ignored. Now we take $d=10 \mu \mathrm{m}$, and interference effects in the PCL, which acts as an anisotropic Fabry-Perot etalon, are accounted for. The overall reflection coefficients of the finite-thickness embedded PCL for incident $p$ - and $s$-polarized light are calculated using equations given in [14].

Figure 5 shows $R_{p}$ and $\mathrm{ER}_{r}$ of the $10 \mu \mathrm{m} \overline{\mathrm{Zn} T e} \mathrm{SWS}$ 1-D PCL embedded in a $\mathrm{ZnS}$ cube as functions of $f$ for $\lambda=10.6 \mu \mathrm{m}, \Lambda=1 \mu \mathrm{m}$, and $\phi=45^{\circ} . R_{p}$ has a flat minimum, $R_{p} \approx 0$, at and near $f=0.604$; the $\mathrm{ER}_{r}$ has a sharp maximum at the same location, and a secondary maximum appears at $f=0.625$. Therefore, excellent performance is still maintained when interference effects in the PCL are considered.

Figure $\underline{6}$ gives the spectral response of the PBS for $p$ - and $s$-polarized light over the broadband $4 \leq \lambda \leq 12 \mu \mathrm{m} . R_{p}$ exhibits interference oscillations but remains $<10^{-6}$; hence the $p$ polarization is essentially totally transmitted. On the other hand, because total internal reflection occurs for $s$-polarized light at the prism-PCL interface, $R_{s}$ falls off monotonically as $\lambda$ increases. This is consistent with the increase in optical tunneling as $d / \lambda$ decreases.

From $R_{p}$ and $R_{s}$ of Fig. 6, $\mathrm{ER}_{r}$ is calculated using Eq. (). Figure $\underline{7}$ shows $E \bar{R}_{r}$ versus $\lambda$ for $d=10 \mu \mathrm{m}$ when interference effects in the PCL are taken into account. Near-ideal polarization on reflection $\left(\mathrm{ER}_{r}>60 \mathrm{~dB}\right)$ is obtained over the $4-12 \mu \mathrm{m}$ IR band. The $\mathrm{ER}_{r}$ shows multiple Fabry-Perot-like peaks at

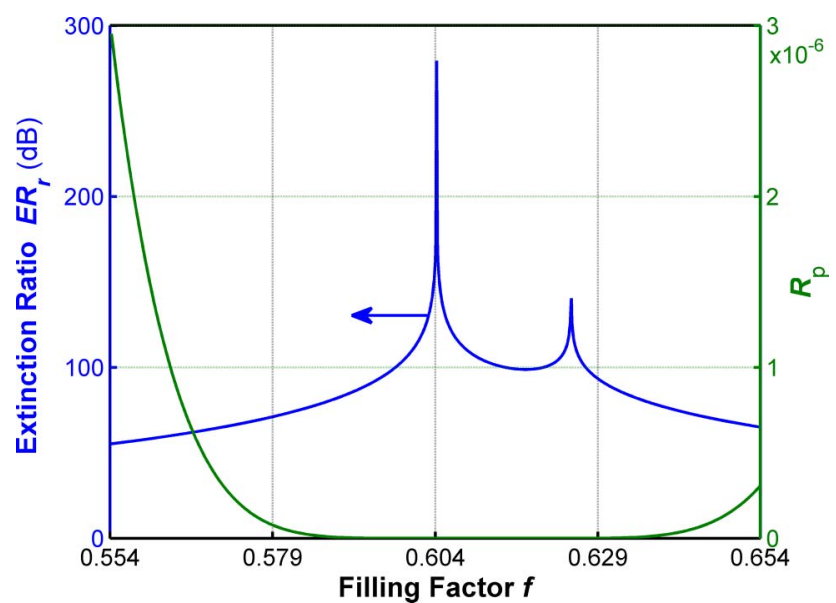

Fig. 5. (Color online) Intensity reflectance $R_{p}$ and extinction ratio $\mathrm{ER}_{r}$ of ZnTe 1-D PCL of thickness $d=10 \mu \mathrm{m}$ embedded in ZnS cube plotted as functions of $f$ for $\lambda=10.6 \mu \mathrm{m}, \Lambda=1 \mu \mathrm{m}$, and $\phi=45^{\circ}$. Interference effects in the PCL are accounted for.



Fig. 6. (Color online) Reflectances $R_{p}$ and $R_{s}$ of ZnTe 1-D PCL of thickness $d=10 \mu \mathrm{m}, f=0.604$, embedded in $\mathrm{ZnS}$ cube, for incident $p$ - and $s$-polarized light, plotted versus wavelength $\lambda$, for $4 \leq \lambda \leq 12 \mu \mathrm{m}$. $R_{p}$ exhibits interference oscillations, whereas $R_{s}$ decreases monotonically as $\lambda$ increases.

wavelengths where $R_{p}=0$, which is expected from Fig. 6 and Eq. (8).

The extinction ratio in transmission is given by

$$
\mathrm{ER}_{t}=10 \log _{10}\left(T_{p} / T_{s}\right)=10 \log _{10}\left(1-R_{p}\right) /\left(1-R_{s}\right) .
$$

Figure 8 shows $\mathrm{ER}_{t}$ as a function of $\lambda . \mathrm{An}^{\mathrm{E}} \mathrm{ER}_{t}>$ $40 \mathrm{~dB}$ is achieved for $4<\lambda<7 \mu \mathrm{m}$, has a maximum near $\lambda=4.8 \mu \mathrm{m}$, and drops monotonically at longer wavelengths. This behavior is consistent with the decrease of $R_{s}$ as $d / \lambda$ decreases as a result of increased optical tunneling.

Higher values of the extinction ratio in transmission are possible if the PCL thickness $d$ is increased so that optical tunneling of the $s$ polarization is

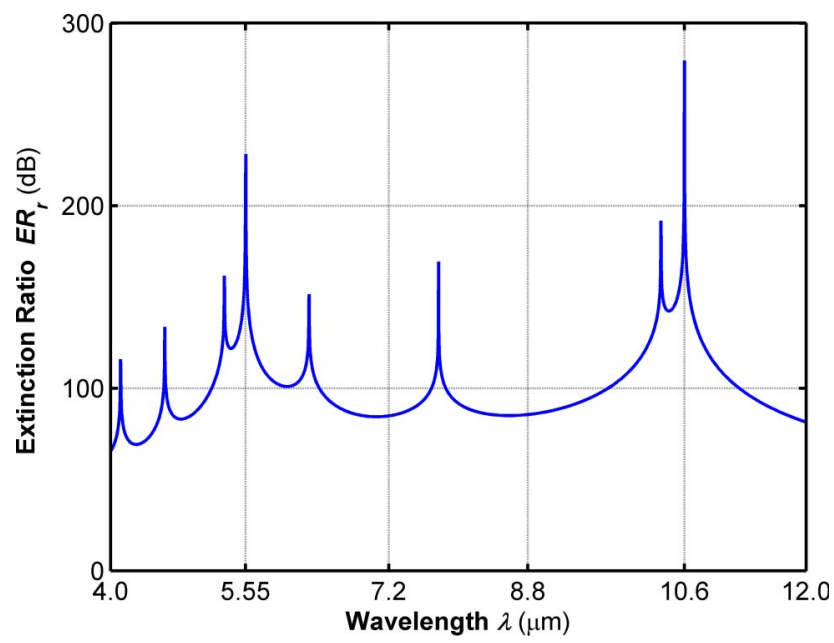

Fig. 7. (Color online) Extinction ratio in reflection $\mathrm{ER}_{r}$ of $\mathrm{ZnTe}$ 1-D PCL of thickness $d=10 \mu \mathrm{m}, f=0.604$, embedded in ZnS cube, shown as a function of wavelength $\lambda$. ER $r$ has Fabry-Perot-type multiple peaks at wavelengths at which destructive interference for the $p$ polarization occurs. 


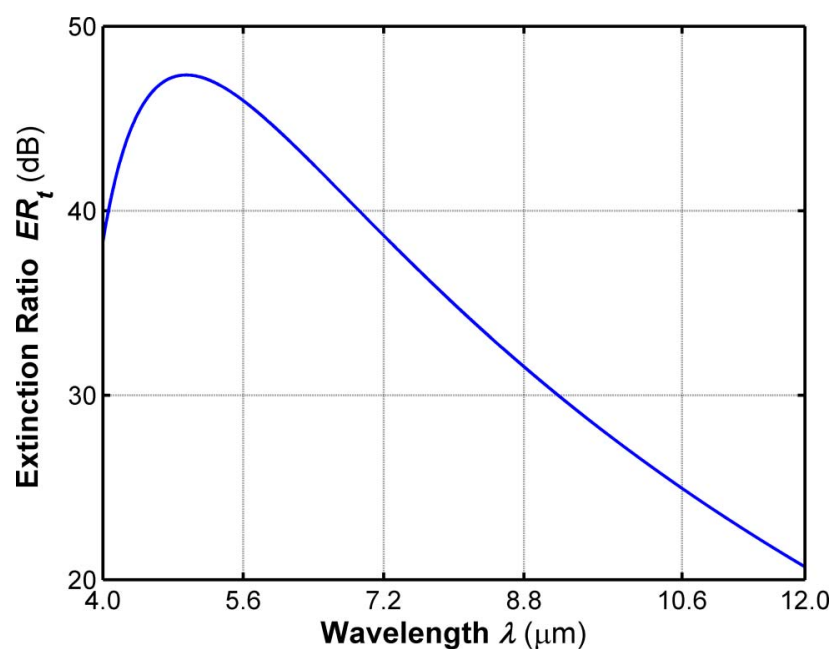

Fig. 8. (Color online) Extinction ratio in transmission $\mathrm{ER}_{t}$, Eq. (9), plotted as a function of wavelength $\lambda$ under the same conditions described in Fig. 7 .

suppressed. Figure $\underline{9}$ shows that $\mathrm{ER}_{t}(\mathrm{~dB})$ is essentially an increasing linear function of $d$, when all other parameters $(f=0.6042, \lambda=10.6 \mu \mathrm{m}$, $\Lambda=1 \mu \mathrm{m}$, and $\phi=45^{\circ}$ ) are kept constant. However, higher values of $d$ pose a technical challenge in device fabrication as the aspect ratio $d / f \Lambda$ of the grating ridges increases.

\section{Polarizing Beam Splitter Using Ge 1-D PCL Embedded in Si Prism}

For a PBS that uses the Ge-Si material system the results are summarized in Table 2 . Table 2 lists the coating and prism refractive indices $\left(n_{c}, \bar{n}\right)$, the ordinary and extraordinary refractive indices $\left(n_{o}, n_{e}\right)$ of the PCL, the critical angle of total internal reflection for the $s$ polarization $\phi_{c s}$, and the grating filling factor $f$ as calculated for $\lambda=10.6 \mu \mathrm{m}$ and $\Lambda=1 \mu \mathrm{m}$. This PCL exhibits giant birefringence, $\Delta n=n_{e^{-}}$ $n_{o}=-1.6445$, and index matching for the $p$ polariza-

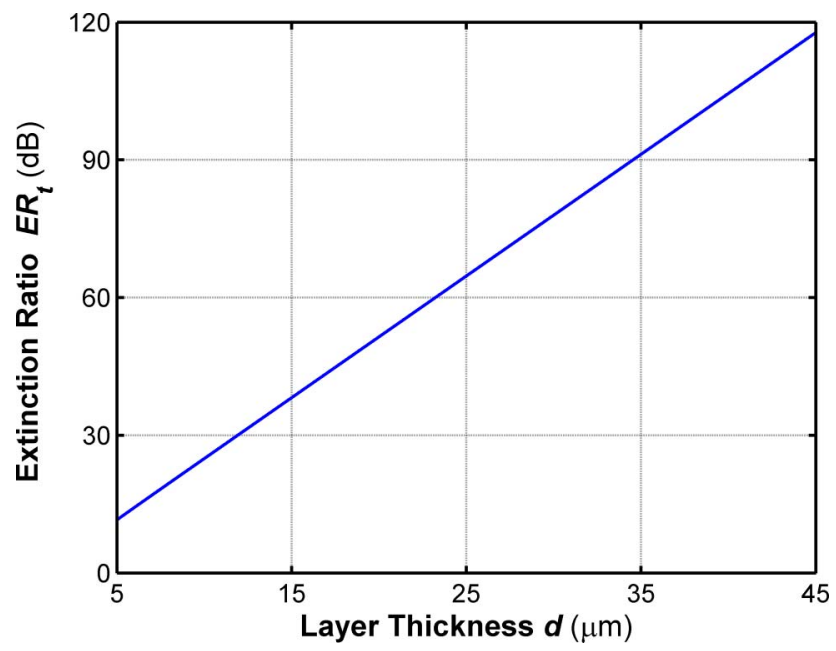

Fig. 9. (Color online) Extinction ratio in transmission $\mathrm{ER}_{t}(\mathrm{~dB})$, Eq. (9), plotted as a function of thickness $d(\mu \mathrm{m})$ for ZnTe 1-D PCL embedded in $\mathrm{ZnS}$ prism with $f=0.604$ and at wavelength $\lambda=10.6 \mu \mathrm{m}$.
Table 2. Design Parameters for PBS Using Ge PCL with $\Lambda=1 \mu \mathrm{m}$ Embedded in Si Cube at $10.6 \mu \mathrm{m}$ Wavelength

\begin{tabular}{cccccc}
\hline$n_{c}(\mathrm{Ge})$ & $f$ & $n_{e}$ & $n_{o}$ & $n(\mathrm{Si})$ & $\phi_{c s}$ \\
\hline 4.0030 & 0.6908 & 1.7731 & 3.4176 & 3.4176 & $31.2528^{\circ}$ \\
\hline
\end{tabular}

tion occurs only at the design wavelength $\lambda=10.6 \mu \mathrm{m}$. As with the $\mathrm{ZnTe}-\mathrm{ZnS}$ design presented in Section 4, this PBS is insensitive to small changes of $f$ around the design value $f=0.691$ and has high values of $\mathrm{ER}_{r}$ and $\mathrm{ER}_{t}$. The major advantage that the Ge-Si material system provides is a much improved internal and external FOV of $\pm 14^{\circ}$ and $\pm 47^{\circ}$, respectively.

\section{Concluding Remarks}

PBSs that use SWS 1-D PCL embedded in a highindex cube prism are demonstrated. Such PBSs are based on index matching and total transmission of the $p$ polarization and total internal reflection of the $s$ polarization at the prism-PCL interface at $45^{\circ}$ angle of incidence. Results obtained for two material systems ( $\mathrm{ZnTe}-\mathrm{ZnS}$ and $\mathrm{Ge}-\mathrm{Si}$ ) show excellent performance over a broad IR spectral range, good field of view, and insensitivity to small errors of grating filling factor. The design procedure is based on the homogenous uniaxial crystal model of the SWS 1-D PCL, and the second-order ordinary and extraordinary refractive indices of that layer are employed. These results are subject to further refinement using rigorous coupled-wave analysis [15]. This extension, device fabrication issues, and the effect of misalignment of the optic-axis direction fall outside the scope of this paper.

\section{References}

1. J. M. Bennett, "Polarization," in Handbook of Optics, M. Bass, E. W. Van Stryland, D. R. Williams, and W. L. Wolfe, eds. (McGraw-Hill, 1995), Vol. I, Chap. 5.

2. H. A. Macleod, Thin Film Optical Filters, 2nd ed. (McGrawHill, 1986).

3. J. Mouchart, J. Begel, and E. Duda, "Modified MacNeille cube polarizer for a wide angular field," Appl. Opt. 28, 28472853 (1989).

4. L. Li and J. A. Dobrowolski, "Visible broadband, wide-angle, thin-film multilayer polarizing beam splitter," Appl. Opt. 35, 2221-2225 (1996).

5. L. Li and J. A. Dobrowolski, "High-performance thin film polarizing beam splitter operating at angles greater than the critical angle," Appl. Opt. 39, 2754-2771 (2000).

6. S. R. Perla and R. M. A. Azzam, "Wide-angle, high-extinctionratio, infrared polarizing beam splitters using frustrated total internal reflection by an embedded centrosymmetric multilayer," Appl. Opt. 46, 4604-4612 (2007).

7. H. Haidner, P. Kipfer, J. T. Sheridan, J. Schwider, N. Streibl, J. Lindolf, M. Collischon, A. Lang, and J. Hutfless, "Polarizing reflection grating beamsplitter for the $10.6 \mu \mathrm{m}$ wavelength," Opt. Eng. 32, 1860-1865 (1993).

8. A. G. Lopez and H. G. Craighead, "Wave-plate polarizing beam splitter based on form-birefringent multilayer grating," Opt. Lett. 23, 1627-1629 (1998). 
9. J. Zheng, C. Zhou, J. Feng, and B. Wang, "Polarizing beam splitter of deep-etched triangular-groove fused-silica gratings," Opt. Lett. 33, 1554-1556 (2008).

10. D. R. Solli, C. F. McCormick, R. Y. Chiao, and J. M. Hickman, "Photonic crystal polarizers and polarizing beam splitters," J. Appl. Phys. 93, 9429-9431 (2003).

11. O. Kilic, S. Fan, and O. Solgaard, "Analysis of guidedresonance-based polarization beam splitting in photonic crystal slabs," J. Opt. Soc. Am. A 25, 2680-2692 (2008).

12. D. L. Brundrett, E. N. Glytsis, and T. K. Gaylord, "Homogeneous layer models for high-spatial-frequency dielectric surface-relief gratings: conical diffraction and antireflection designs," Appl. Opt. 33, 2695-2706 (1994).

13. W. J. Tropf, M. E. Thomas, and T. J. Harris, "Properties of crystals and glasses," in Handbook of Optics, M. Bass, E. W. Van Stryland, D. R. Williams, and W. L. Wolfe, eds. (McGraw-Hill, 1995), Vol. II, Chap. 33.

14. R. M. A. Azzam and N. M. Bashara, Ellipsometry and Polarized Light (North-Holland, 1987), Section 4.7.3.

15. M. G. Moharam and T. K. Gaylord, "Diffraction analysis of dielectric surface-relief gratings," J. Opt. Soc. Am. 72, 13851392 (1982). 